Supporting information for

\title{
Transport Dynamics of Neutral Excitons and Trions in Monolayer $\mathbf{W S}_{2}$
}

\author{
Toshiaki Kato* \& Toshiro Kaneko
}

Department of Electronic Engineering, Tohoku University, 980-8579 Sendai, Japan

\section{Contents}

[1] Fabrication process of suspended TMD device

[2] Effects of excitation power on the PL measurement

[3] Measurement of exciton diffusion length with CCD imaging

[4] Activation energy for ionized impurity scattering

[5] Trion diffusion length measured by size dependent PL intensity 


\section{[1] Fabrication process of suspended TMD device}

The detailed fabrication process of suspended TMD was shown in Fig. S1. The electrode with different gap was formed with a conventional electron beam lithography and vacuum deposition of $\mathrm{Au}$ metal prior to the lift off process. The monolayer $\mathrm{WS}_{2}$ and $\mathrm{WSe}_{2}$ were obtained by mechanical exfoliation and transferred to the electrodes by home-made micro position control unite combined with microscope.

(a)

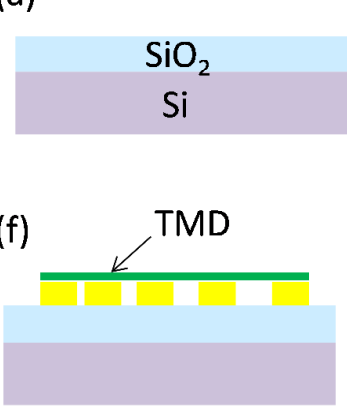

(b)

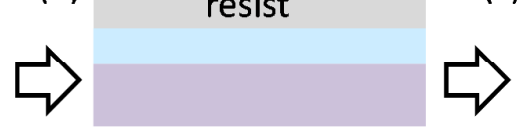

(c)

(e)

(d)
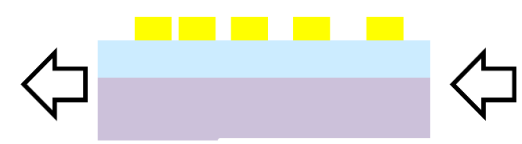

(h)

(g)

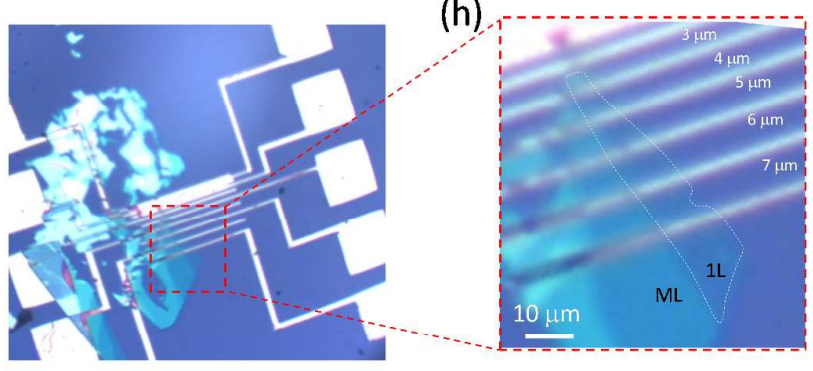

Figure S1: (a-f) Schematic illustration of fabrication process of suspended $\mathrm{WS}_{2}$. $(\mathrm{a}, \mathrm{b})$ The electron beam resist was spin coated on a thermally oxidized $\mathrm{SiO}_{2} / \mathrm{Si}$ substrate (a). (c-e) The electrode pattern was drawn by electron beam lithography (c) and Au film was deposited (d) to form the electrode pattern with different electrode distance between 3 to $7 \mu \mathrm{m}$ (e). TMD was transferred on the top of electrodes to form the suspended structures (f). (g,h) Typical optical microscope images of suspended $\mathrm{WS}_{2}$ device with low (g) and high (h) magnification images. 


\section{[2] Effects of excitation power on the PL measurement}

The $I_{\mathrm{PL}}$-power plot of the excitons is shown in Fig. S2, which indicates that the laser power used in this measurement $(0.5 \mathrm{~mW})$ is within the linear dependence of the $I_{\mathrm{PL}}$-power plot. This suggests that the density of excitons in $\mathrm{WS}_{2}$ is low, and that exciton-exciton annihilation is negligible in this measurement. These results support the accuracy of the diffusion model used in this study to estimate the exciton diffusion length.

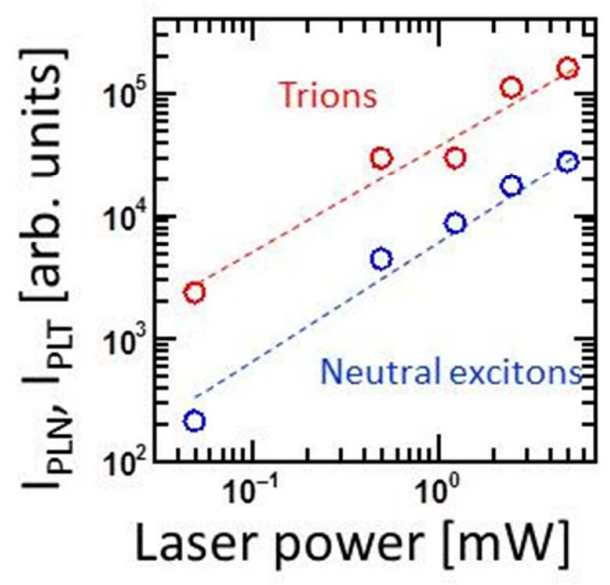

Figure S2: Excitation laser power dependence of $I_{\text {PLN }}$ and $I_{\text {PLT. }}$ 


\section{[3] Measurement of exciton diffusion length with CCD imaging}

The exciton diffusion length was measured directly by CCD imaging. The detailed analysis process is as follows. The CCD image is decomposed into raw PL spectra for different special positions (Fig. S3(a, b). Spectral fitting is carried out for each raw spectrum, decomposing it into neutral exciton and trion components (Fig. S3(c)). Then the integrated intensity of the neutral exciton obtained by fitting is plotted as a function of distance from the excitation center, obtaining the spatial distribution of neutral exciton density (Fig. S3(d)). The exciton diffusion length can then be obtained Fig. S3(d), by numerically solving the exciton density profile. As mentioned in the main text, the spatial distribution of excitons from the excitation center can be calculated by numerically solving

$$
L_{d i f}^{2} \frac{d^{2} n(x, y)}{d x^{2}}+L_{d i f}^{2} \frac{d^{2} n(x, y)}{d y^{2}}-n(x, y)+N \exp \left(-\frac{x^{2}+y^{2}}{\sigma^{2}}\right)=0
$$

where $L_{\mathrm{dif}}\left(=(D \tau)^{1 / 2}\right)$ and $N\left(=\Gamma_{0} \tau\right)$ are the exciton diffusion length and constant, respectively. Then, the one-dimensional distribution profile of the exciton can be obtained by taking the exciton density on the line shown in Fig. S3(e) (from (i) to (ii)) (Fig. S3(f)). By changing $L_{\text {dif }}$ in equation (S1), we obtained the 1/e spatial distribution length as a function of $L_{\mathrm{dif}}$, which is plotted in Fig. S3(g). Finally, $L_{\mathrm{N}}$ was obtained 
through a comparison of the experimentally obtained 1/e spatial distribution length (Fig.

S3(d)) and the calculated results (Fig. S3(f)).

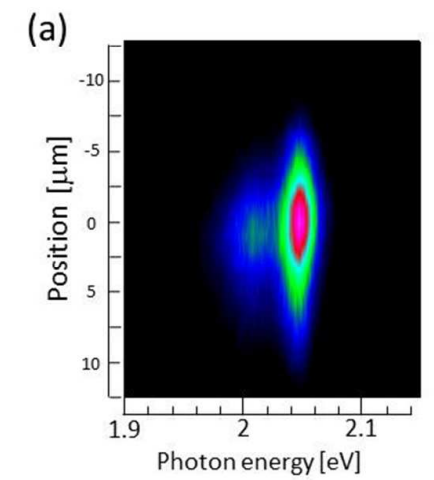

(c)

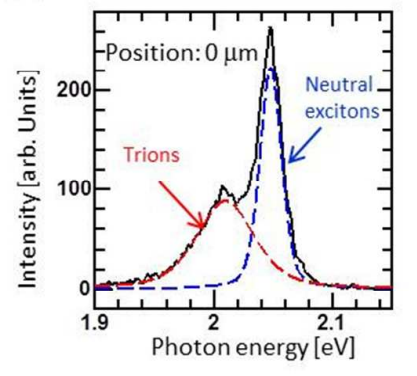

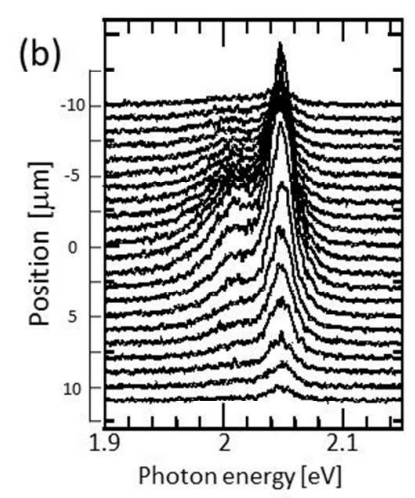

(d)

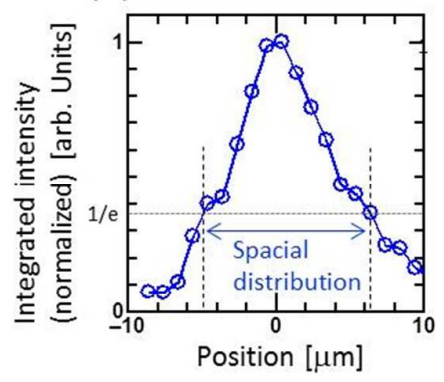

(e)
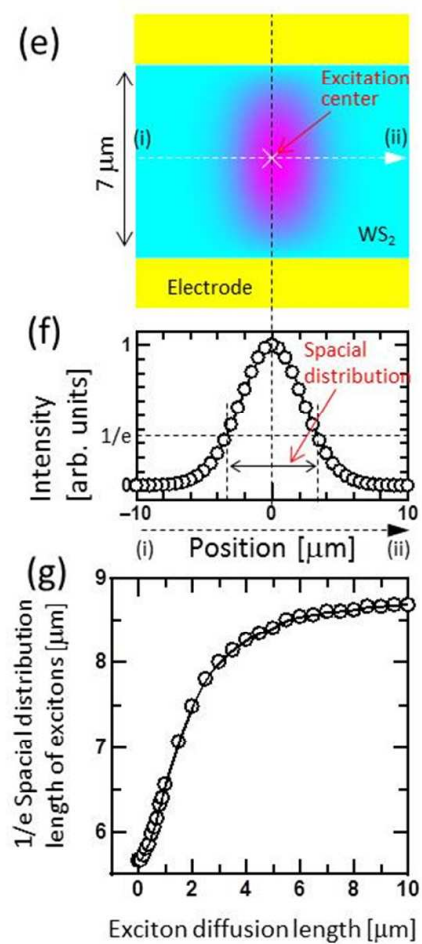

Figure S3: Method for direct observation of diffusion length by CCD imaging. (a) Typical CCD image, (b) decomposed spectra from (a), and (c) typical raw PL spectrum measured from a suspended monolayer $\mathrm{WS}_{2}$ crystal. The blue and red curves in (c) show the fitting curves of neutral exciton and trion components, respectively. (d) Typical spatial profile of the integrated neutral exciton intensity. (e) $2 \mathrm{D}$-spatial profile and (f) $1 \mathrm{D}$ profile of the exciton density (i.e., intensity) obtained by a numerical calculation with a fixed diffusion length. (g) 1/e spatial distribution length as a function of the exciton diffusion length estimated from the calculation data (e, f). 


\section{[4] Activation energy for ionized impurity scattering}

The PL intensity of neutral excitons $\left(I_{\mathrm{PLN}}\right)$ is plotted as a function of $1 / T$ (Fig.

S4). In the middle temperature range $(75 \leq T \leq 200 \mathrm{~K}), \log \left(I_{\mathrm{PLN}}\right)$ increases linearly with decreasing $1 / T$, indicating that $I_{\mathrm{PLN}}$ can be shown with an Arrhenius type plot. Through fitting in the linear correlation region, it is found that the activation energy is about 22 meV.

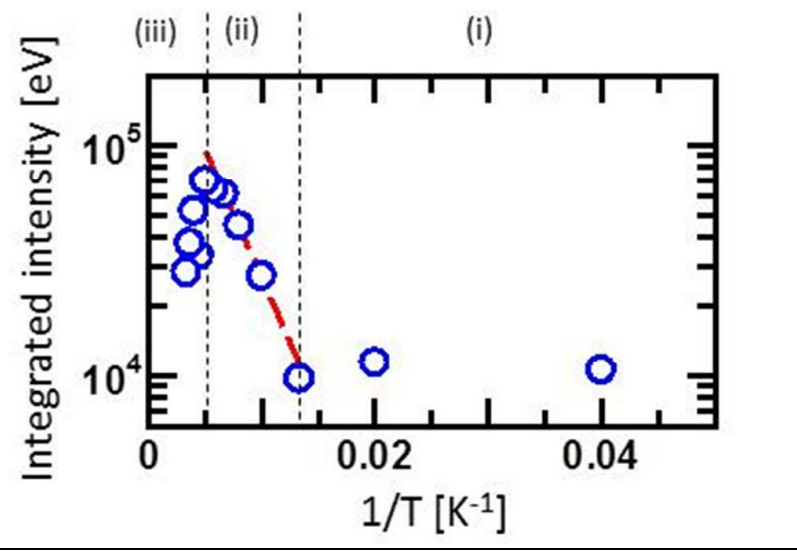

Figure S4: Arrhenius plot of integrated intensity of neutral excitons. The red line shows the fitting curve in region (ii) obtaining the activation energy $(22.09 \mathrm{meV})$. 


\section{[5] Trion diffusion length measured by the size dependent PL intensity}

Diffusion difference between trion and neutral excitons was carried out for suspended $\mathrm{WS}_{2}$ and non-suspended (supported on a $\mathrm{SiO}_{2}$ ) $\mathrm{WS}_{2}$ (Fig. S5(a)). The longer diffusion of trion can be observed only with suspended sample at low temperature range, indicating the impurity free structure should be critical to realize the longer diffusion of trions.

Temperature dependence of normalized D for trion is plotted in Fig. S5(b). The experimental data can be well fitted by $\mathrm{T}^{-1 / 2}$ curve, which can explain due to the acoustic phono scattering.
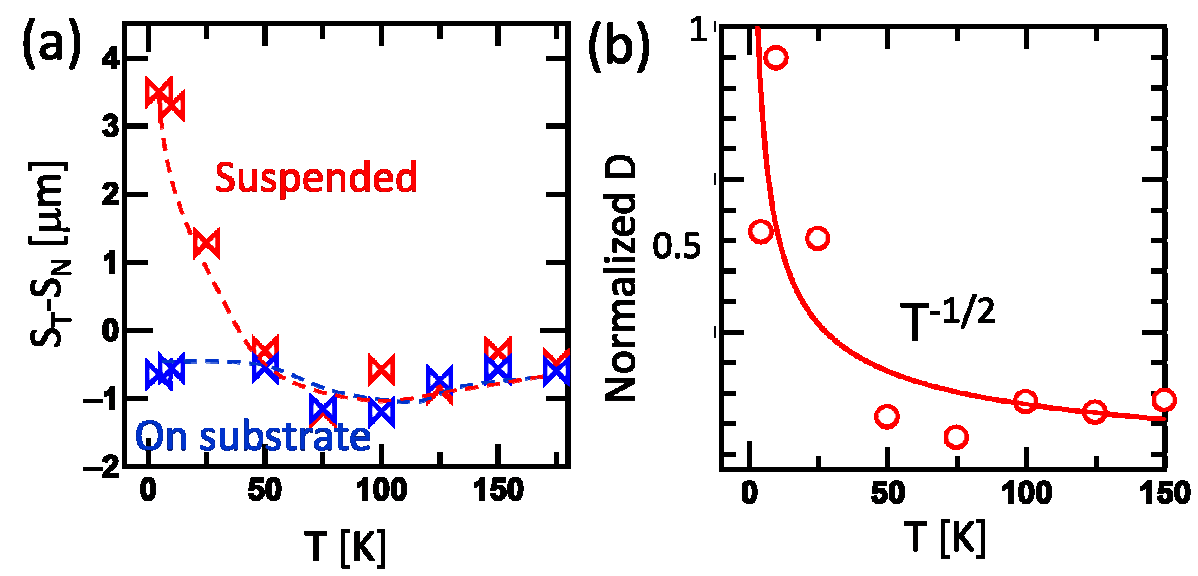

Figure S5: (a) $\mathrm{S}_{\mathrm{T}}-\mathrm{S}_{\mathrm{N}}$ plot as a function of $\mathrm{T}$. The red and blue curves show suspended and non-suspended (supported on a $\mathrm{SiO}_{2}$ surface) $\mathrm{WS}_{2}$, respectively. (b) $\mathrm{T}$ dependence of normalized D of trions. 

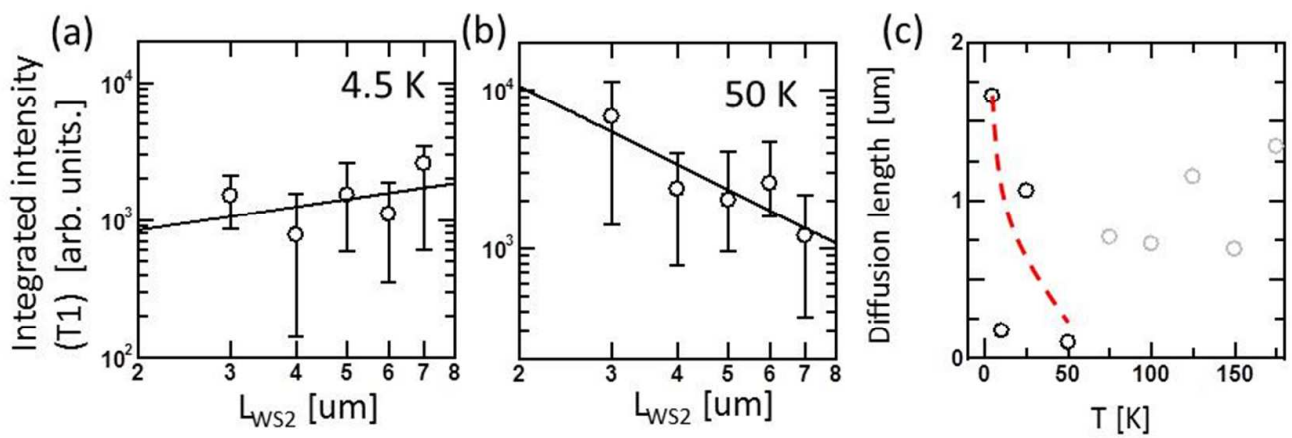

Figure S6: Integrated intensity of trions as a function of $L_{\mathrm{wS} 2}$ measured at (a) $4.5 \mathrm{~K}$ and (b) $50 \mathrm{~K}$. (c) Diffusion length of the trions estimated from the size-dependent PL intensity as a function of $T$.

A size-dependent $I_{\text {PL }}$ analysis was also carried out to estimate the diffusion length of the trions. In the case of $4.5 \mathrm{~K}, I_{\mathrm{PL}}$ of trions (IPLT) increases with the $\mathrm{WS}_{2}$ size (Lws2) (Fig. S6(a)), whereas it decreases with $L$ ws2 at $50 \mathrm{~K}$ (Fig. S6(b)). This indicates that the diffusion length of the trions at $4.5 \mathrm{~K}$ is longer than that at $50 \mathrm{~K}$, as discussed in Figs. 1(b) and 1(c) in the main text. Based on the fitting analysis, it is found that the $L_{\text {dift }}$ reaches about $1.7 \mu \mathrm{m}$, which is much longer than that of neutral excitons in the same temperature range obtained from the same analysis (Fig. S6(c)). These results are consistent with those obtained from the direct CCD imaging shown in the main text. 